\title{
The effect of primary site, functional status and treatment modality on survival in gastroenteropancreatic neuroendocrine neoplasms with synchronous liver metastasis: a US population-based study
}

\author{
Ning Pu ${ }^{1,2 \#}$, Joseph R. Habib ${ }^{2 \#}$, Michael Bejjani ${ }^{3}$, Hanlin Yin ${ }^{1}$, Minako Nagai ${ }^{2,4}$, Jianan Chen ${ }^{2,5}$, \\ Benedict Kinny-Köster ${ }^{2}$, Qiangda Chen ${ }^{1}$, Jicheng Zhang ${ }^{1}$, Jun $\mathrm{Yu}^{2}$, Wenchuan $\mathrm{Wu}^{1}$, Wenhui Lou ${ }^{1}$ \\ ${ }^{1}$ Department of General Surgery, Zhongshan Hospital, Fudan University, Shanghai, China; ${ }^{2}$ Department of Surgery, The Sol Goldman Pancreatic \\ Cancer Research Center, The Johns Hopkins University School of Medicine, Baltimore, MD, USA; ${ }^{3}$ Department of Gastroenterology and \\ Hepatology, The Johns Hopkins University School of Medicine, Baltimore, MD, USA; ${ }^{4}$ Department of Surgery, Nara Medical University, Nara, \\ Japan; ${ }^{5}$ Department of Colorectal Surgery, National Cancer Center/Cancer Hospital, Chinese Academy of Medical Sciences and Peking Union \\ Medical College, Beijing, China \\ Contributions: (I) Conception and design: N Pu, JR Habib, W Lou; (II) Administrative support: W Lou; (III) Provision of study materials or patients: \\ N Pu, JR Habib, W Lou; (IV) Collection and assembly of data: N Pu, JR Habib, M Bejjani, H Yin, M Nagai, J Chen, B Kinny-Köster, Q Chen, J \\ Zhang, J Yu; (V) Data analysis and interpretation: N Pu, JR Habib, W Lou; (VI) Manuscript writing: All authors; (VII) Final approval of manuscript: \\ All authors. \\ \#These authors contributed equally to this work. \\ Correspondence to: Wenhui Lou, MD, PhD. Department of General Surgery, Zhongshan Hospital, Fudan University, 180 Fenglin Road, Shanghai \\ 200032, China. Email: lou.wenhui@zs-hospital.sh.cn.
}

Background: The incidence of indolent gastroenteropancreatic neuroendocrine neoplasms (GEP-NENs) has increased dramatically. GEP-NENs often present late with concomitant liver metastasis, which is associated with poorer outcomes.

Methods: This is a retrospective cohort study of 3,188 patients with liver metastatic GEP-NENs from the national scale Surveillance, Epidemiology, and End Results (SEER) database in the USA between 2010 and 2016. The population-based sample of GEP-NENs with liver metastasis was stratified by primary site (intestinal, pancreatic or gastric), surgical intervention and functional status.

Results: Of the 3,188 patients with liver metastatic GEP-NENs in this study, intestinal NENs (iNENs) were the most common and displayed the best 5 -year survival of $42.6 \%$ compared to $25.8 \%$ in pancreatic NENs (pNENs) and 12.0\% in gastric NENs (gNENs). Surgical intervention [hazard ratio (HR): 0.46, 95\% CI: (0.40-0.53), $\mathrm{P}<0.001]$ and carcinoid subtype showed robust survival advantages across all groups. pNENs with liver metastasis were associated with the greatest benefit of surgery [HR: $0.55,95 \%$ CI: (0.41-0.75), $\mathrm{P}<0.001]$ while iNENs were the most commonly treated by surgery. After risk adjustment, primary site was not associated with outcome in the non-surgical group.

Conclusions: Taken collectively, when diagnosed with liver metastasis, iNENs conferred a better overall prognosis than both pNENs and gNENs. Primary surgical resection, especially of carcinoid type tumors, emerged as a robust prognostic indicator of better outcomes irrespective of primary site. This finding was most pronounced in liver metastatic pNENs. When possible, we recommend surgical intervention in GEPNENs with liver metastasis.

Keywords: Gastroenteropancreatic neuroendocrine neoplasm (GEP-NEN); primary site; functional status; surgical intervention; prognosis

Submitted Jul 22, 2020. Accepted for publication Oct 30, 2020.

doi: $10.21037 /$ atm-20-5348

View this article at: http://dx.doi.org/10.21037/atm-20-5348 


\section{Introduction}

Neuroendocrine neoplasms (NENs), a heterogeneous group of neoplasms originating from the neuroendocrine system, are relatively rare tumors estimated at around 6.8 per 100,000 in the US population $(1,2)$. In the past two decades, the reported incidence of NENs has increased, likely due to advancements in radiographic diagnostic techniques and awareness. NENs most commonly arise in the gastrointestinal system, where they are collectively referred to as gastroenteropancreatic neuroendocrine neoplasms (GEP-NENs) $(3,4)$.

GEP-NENs occur in two major tumor sub-types (carcinoid and non-carcinoid), and generally follow an indolent course that often results in affected patients presenting late with established metastatic disease, predominantly in the liver (5). Liver metastasis has been estimated to account for up to $82 \%$ of all GEP-NEN metastases, and about $40 \%$ of patients with GEP-NENs will develop liver metastasis during their clinical course $(6,7)$. Unsurprisingly, such liver metastases cause progressive liver failure and worse survival rates (8). These collective characteristics of GEP-NENs largely determine the choice of primary treatment modality (surgical resection versus non-surgical), which remains particularly controversial in case of associated liver metastasis (9).

Studies investigating the potential prognostic role of the primary site of GEP-NENs are limited. One study showed that GEP-NENs of primary pancreatic origin are associated with poor survival while another identified NENs originating in the rectum to have relatively more favorable survival $(3,10)$. More specifically, reports on the prognostic role of organ primary site and its interaction with the roles of both functional status and primary treatment modality choice in the setting of GEP-NENs with synchronous liver metastasis are lacking. In this study, we aimed to leverage the available national scale patient survival data from the Surveillance, Epidemiology, and End Results (SEER) database to systematically elucidate these interactive prognostic roles of primary site, functional status and treatment modality in liver metastatic GEP-NENs in the USA. We present the following article in accordance with the Strengthening the Reporting of Observational studies in Epidemiology (STROBE) reporting checklist (available at http://dx.doi.org/10.21037/atm-20-5348).

\section{Methods}

Access to the database for research purposes was approved by the SEER Committee. SEER*Stat 8.3.6 software was used to extract data from the SEER database [IncidenceSEER 18 Regs Custom Data (with additional treatment fields), Nov. 2018 Sub (1975-2016 varying)] as previously reported (11). The study was conducted in accordance with the Declaration of Helsinki (as revised in 2013). The study was approved by institutional ethics committee of Zhongshan Hospital, Fudan University (No. Y2020.094) and individual consent was waived due to the retrospective nature of the analysis.

\section{Study population}

Patients with a diagnosis of primary GEP-NEN were identified from the SEER database, for the period between 2010 and 2016, based on the primary tumor site and morphology data fields (Figure S1). First, patient records with GEP tumors were identified based the site data field (CD-O-3/WHO 2008) and included the following: Stomach, Small Intestine, Colon and Rectum, Colon excluding Rectum, Cecum, Appendix, Ascending Colon, Hepatic Flexure, Transverse Colon, Splenic Flexure, Descending Colon, Sigmoid Colon, Large Intestine NOS, Rectum and Rectosigmoid Junction, Rectosigmoid Junction, Rectum, Anus, Anal Canal and Anorectum and Pancreas. GEP tumors were next restricted to the following NENs defined according to the International Classification of Diseases for Oncology (3rd edition) for tumor of histology/ behavior, and included the following: pancreatic endocrine tumor, malignant $(8,150 / 3)$, insulinoma, malignant $(8,151 / 3)$, glucagonoma, malignant $(8,152 / 3)$, gastrinoma, malignant $(8,153 / 3)$, mixed pancreatic endocrine and exocrine tumor, malignant $(8,154 / 3)$, vipoma, malignant $(8,155 / 3)$, somatostatinoma, malignant $(8,156 / 3)$, carcinoid tumor $(8,240 / 2)$, enterochromaffin cell carcinoid $(8,241 / 3)$, enterochromaffin-like cell tumor, malignant $(8,242 / 3)$, goblet cell carcinoid $(8,243 / 3)$, mixed adenoneuroendocrine carcinoma $(8,244 / 3)$, adenocarcinoid tumor $(8,245 / 3)$, neuroendocrine carcinoma in situ $(8,246 / 2)$, neuroendocrine carcinoma, NOS $(8,246 / 3)$ and atypical carcinoid tumor $(8,249 / 3)$.

\section{Study groups}

For the current analysis, GEP-NENs were analyzed both collectively accounting for treatment approach, and after stratification to treatment modality sub-cohorts (primary surgical resection: Yes or No). In all cases, the primary sub- 
group comparisons were focused on primary site (intestine, pancreas and stomach), and with each further subdivided based on functional status (carcinoid or non-carcinoid). Cases were excluded in case the data record included evidence of other malignancies or other unknown metastatic locations outside the liver.

\section{Patient factors}

Demographic and clinicopathologic risk factors considered as covariates in this analysis included the following: gender, age, primary site, tumor grade, functional status, primary surgical resection, chemotherapy, radiotherapy and distal metastasis (bone, lung and brain). The number of examined (ELN) and positive lymph nodes (PLN) and their ratio $[$ lymph node ratio $(\mathrm{LNR})=\mathrm{PLN} / \mathrm{ELN}]$ were also considered in a secondary analysis in the surgical GEP-NEN sub-cohort. Survival was compared across increasing PLN groups for all GEP-NEN origin/location groupings in accordance to the 8th edition of the American Joint Committee on Cancer's (AJCC) tumor, nodes, and metastases, (TNM) staging system for pancreatic cancer $[\mathrm{N}$ classification groups: N0 (no PLN), N1 $(1 \leq \mathrm{PLN} \leq 3)$ and N2 (PLN $\geq 4)]$.

\section{Statistical analysis}

Statistical analyses were conducted using $\mathrm{R}$ project version 3.3.3 (http://www.r-project.org/) and SPSS 21.0 statistical package (SPSS Inc., Chicago, IL, USA) for Windows. Continuous variables were compared using the MannWhitney U test or Kruskal-Wallis test, as appropriate. Chi-squared or Fisher's exact tests (if needed) were used for categorical variables when appropriate. Longitudinal follow-up data were analyzed to determine survival using Kaplan-Meier analysis with log-rank test between-group comparisons. Univariate and multivariate Cox proportional hazard regressions were used to estimate unadjusted (HR) and risk adjusted hazard ratios (AHR), respectively. A P value $<0.05$ was used to indicate statistical significance of all independent prognostic factors.

\section{Results}

\section{Study population and comparison groups}

The SEER database, between 2010 and 2016, was comprised of a total of 30,727 patients diagnosed with
GEP-NENs. Synchronous liver metastasis was prevalent in 4,225 of these patients. Patients with unknown metastatic disease in locations other than the liver $(n=164)$ and those with other types of primary malignancies $(n=874)$ were excluded for a final study population of 3,188 patients (Figure S1).

Patient and clinicopathologic factors for the study population are detailed in Table 1 . These were distinctly different between the surgical resection versus the nonsurgical sub-cohorts (Table 1). The intra-organ location of the primary tumor within each of the tumor origin site (intestine, pancreas, and stomach) for the entire study population are detailed in Figure 1. Briefly, tumors originating in the intestine (iNENs: $n=1,590,49.87 \%$ ) were the most common, followed by pancreas-derived tumors (pancreatic NENs, pNENs: $n=1,427,44.76 \%$ ), while gastric-originating tumors (gastric NENs, gNENs: $n=171$, $5.36 \%)$ were relatively infrequent. When specified, the majority of pNENs had their intra-organ tumor originating in the tail $(n=407,30.69 \%)$, followed by the head $(n=409$, $28.66 \%)$ and then body $(n=155,10.86 \%)$. For gNENs, the primary intra-organ location was most commonly in the cardia $(n=46,26.90 \%)$ and rarest in the pylorus $(n=2$, $1.17 \%)$. For iNENs, 614 (38.62\%) cases originated from the colorectal region while 457 (28.74\%) originated from the ileum (Figure 1).

The primary treatment approach in this SEER GEPNEN with synchronous liver metastasis patient series was more frequently non-surgical (1,959 of 3,$188 ; 61.4 \%$ ) versus involving primary surgical resection in 1,204 patients (37.8\%). Notably, the rate of surgical intervention rate was nearly four-fold greater in case of iNENs (947 of 1,590; $59.6 \%$ ) compared to both pNENs (232 of 1,$427 ; 16.3 \%$ ) and gNENs (25 of $171 ; 14.6 \%$; Table 1$)$.

Univariate analysis performed on all GEP-NENs with synchronous liver metastasis cases showed that gender, age, primary tumor site, tumor grade, functional status, surgical resection (Yes/No), radiotherapy, chemotherapy and synchronous bone, brain or lung metastasis were all significantly associated with survival (Table 2, Table S1).

\section{Observed survival in GEP-NENs with synchronous liver metastasis and the role of tumor origin}

The observed unadjusted survival of GEP-NENs with synchronous liver metastasis differed significantly across the three tumor origin groups (Figure $2 A ; \mathrm{P}<0.001 \log$ rank). Overall, iNEN patients were associated with the 
Table 1 Clinicopathologic factors and their correlations with surgical resection in all GEP-NEN patients with liver metastasis (SEER 2010-2016)

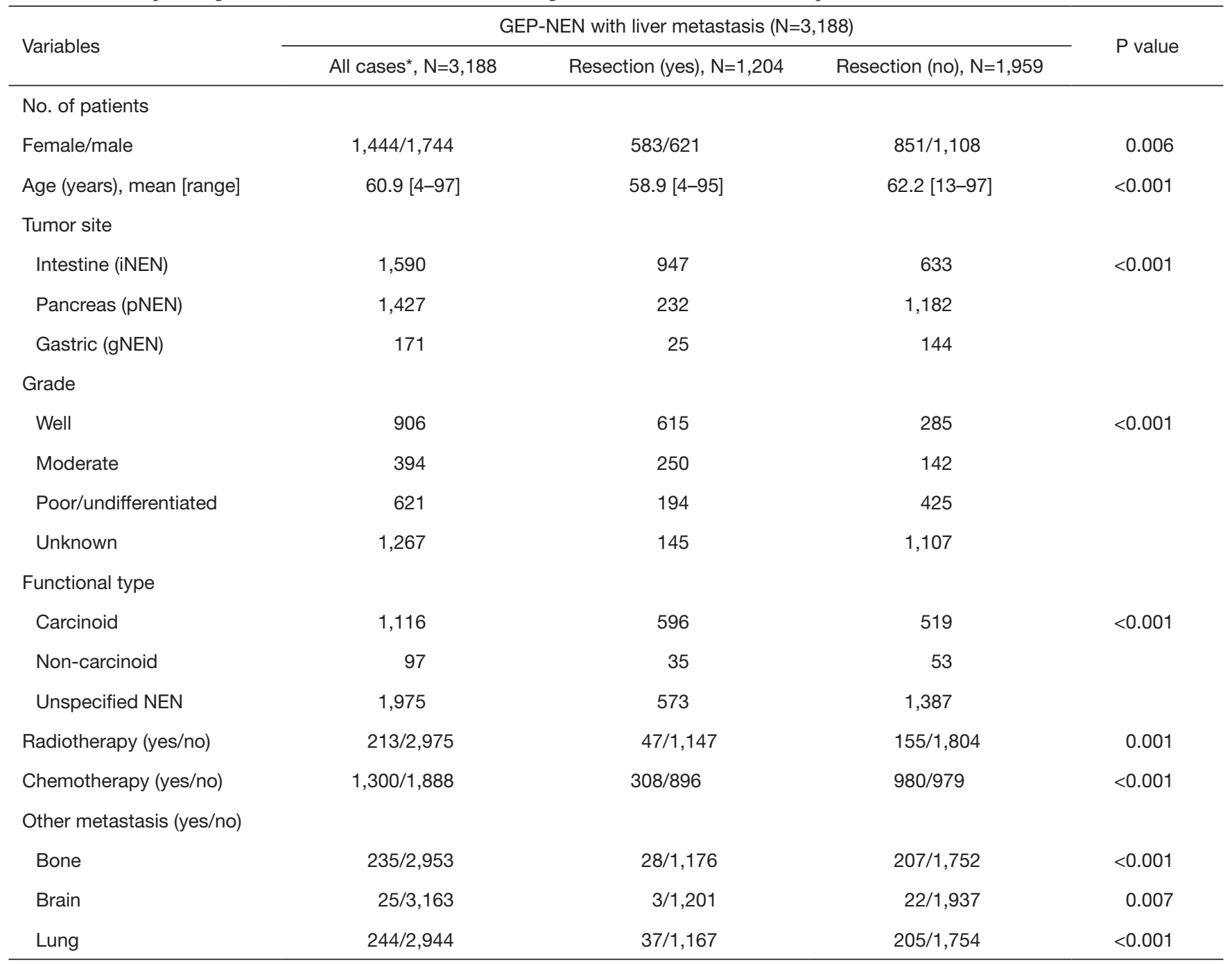

*, all cases include 25 patients with unknown surgical resection status. GEP-NEN, gastroenteropancreatic neuroendocrine neoplasms; SEER, Surveillance, Epidemiology, and End Results.

best unadjusted median (95\% CI) survival [43 (37.248.8) months], pNEN patients exhibited intermediate survival [22 (19.4-24.6) months] and gNEN patients were associated with the worst survival [8 (5.5-10.5) months]. The 5 -year survival rates were $42.6 \%, 25.8 \%$ and $12.0 \%$, for iNENs, pNENs and gNENs, respectively (Figure $2 A$ ). The corresponding unadjusted hazard ratios for primary tumor site are summarized in Table 2. However, primary resected pNEN patients had the best survival, resected iNEN patients exhibited intermediate survival, and resected gNEN patients were associated with the worst survival (1-year survival rate of $90.6 \%, 81.2 \%$ and $64.7 \%$ respectively; 3 -year survival rate of $74.7 \%, 69.5 \%$ and
$54.7 \%$ respectively, Figure 2B).

Considering surgical and non-surgical cases combined (Figure $2 C, D, E, F)$, iNEN patients exhibited a large degree of survival variation depending on the specific tumor site within the intestinal tract. Median survival in case of proximal intestinal origins was significantly better than for cases with distal intestinal location such as colon or rectum origins (Figure $3 A$ ). Moreover, a significant degree of variability existed even within the proximal intestine where tumors originating from the ileum and jejunum conferred better survival than the duodenum (Figure 3B). In contrast, there was no significant survival difference in case of pNENs whether originating in the head, body or tail (Figure 3C) or 


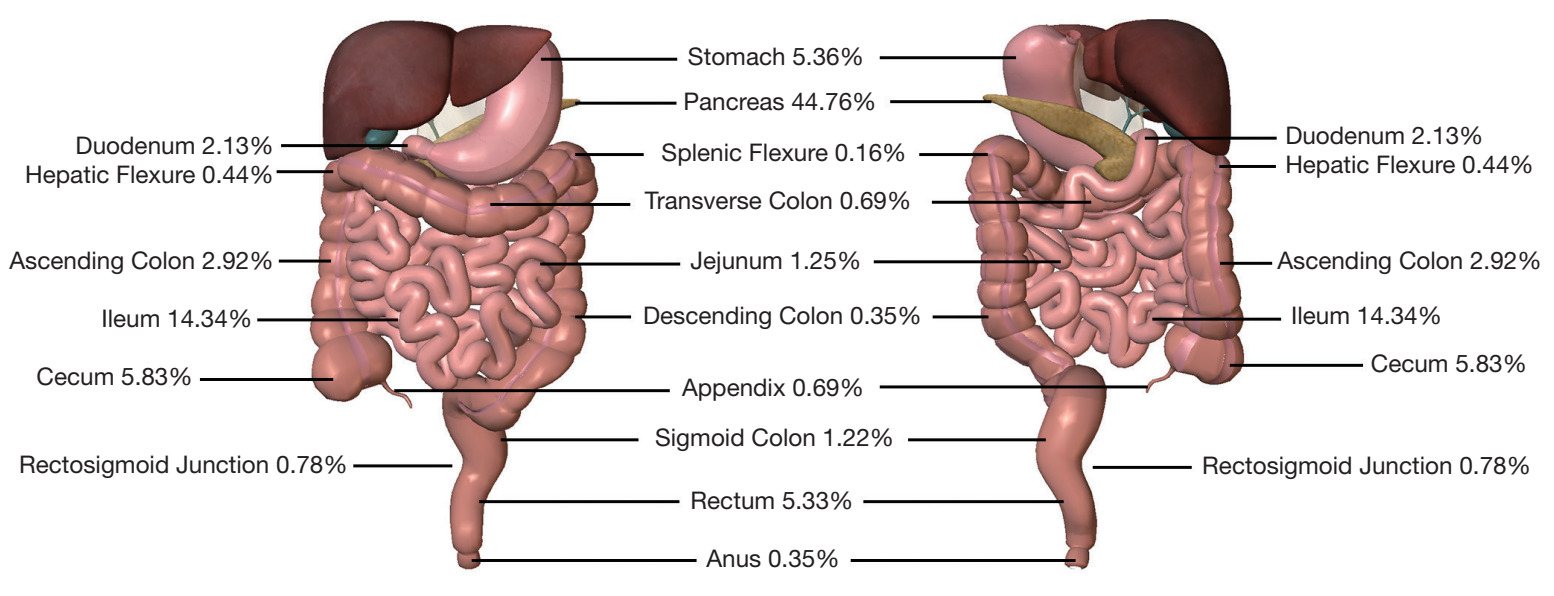

Small Intestine, NOS $11.86 \%$

Large Intestine, NOS 1.54\%
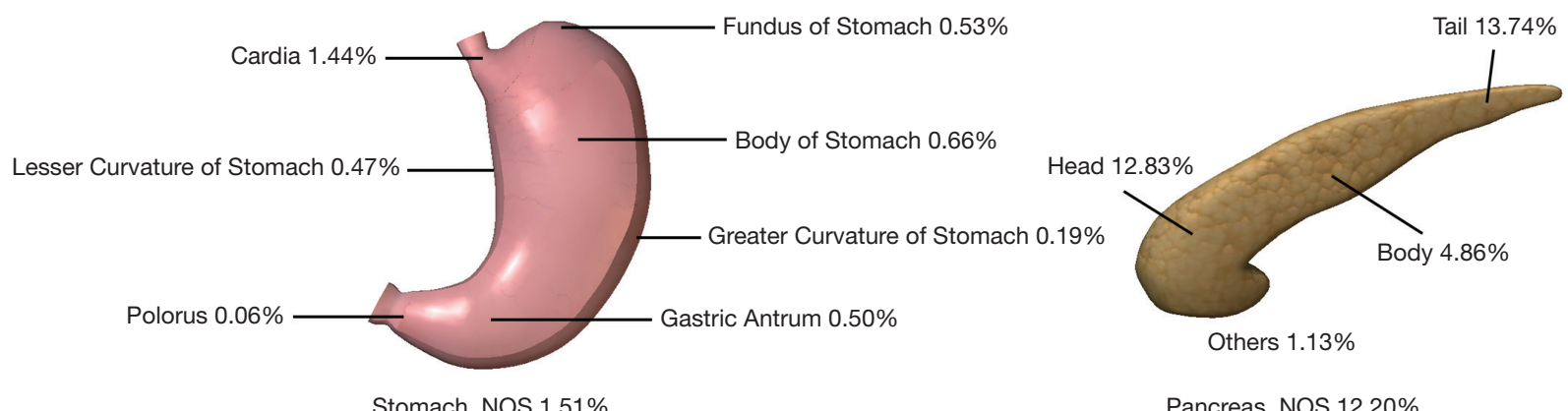

Stomach, NOS $1.51 \%$

Pancreas, NOS $12.20 \%$

Figure 1 The distribution of GEP-NENs with synchronous liver metastasis amongst the primary origins. The anatomical images were picked from Atlas software. GEP-NEN, gastroenteropancreatic neuroendocrine neoplasms.

in case of different locations within the stomach (Figure 3D).

\section{Observed survival in GEP-NENs with synchronous liver metastasis and the role of treatment modality}

GEP-NEN with synchronous liver metastasis patients were generally associated with a substantially superior survival in case of primary surgical resection [1-year: $82.7 \%, 3$-year: $70.2 \%, 5$-year: 59.2\%] compared to non-surgical treatment [1-year: $52.5 \%$, 3-year: $28.5 \%, 5$-year: $16.8 \%, \mathrm{P}<0.001$; Figure $2 C$ ). The corresponding unadjusted hazard ratios for both treatment modalities are summarized in Table 2 . Importantly, the large difference in survival between surgical resection (better) versus non-surgical (worse) patients was true in case of intestinal, pancreas and stomach tumor origin albeit to different degrees (Figure 2D,E,F; all $\mathrm{P}<0.001)$. In non-surgical patients, survival was comparable for all three tumor origin subgroups.

Lymph node pathology examination data were available in 1,022 of the $1,204(85 \%)$ patients treated with surgical resection. The median LNR was lowest for pNEN cases (LNR $=0.154)$, intermediate in gNENs ( $\mathrm{LNR}=0.218)$, and highest for iNEN patients (LNR $=0.308$ ). The LNR was highest in case of distal intestinal GEP-NEN with median LNR of 0.333 and 0.5 for appendiceal and colorectal/anal NENs, respectively $(\mathrm{P}<0.001)$. Increasing PLN staging was associated with significantly worse survival in case of pNENs $(\mathrm{P}=0.010$, Figure $4 A)$ and colorectal/anal NENs $(\mathrm{P}=0.005$, Figure $4 B)$, but not in the case of gastric $(\mathrm{P}=0.151$, Figure $4 C)$, small intestinal $(\mathrm{P}=0.059$, Figure $4 D)$ or appendiceal NENs $(\mathrm{P}=0.074$, Figure $4 E)$.

\section{Observed survival of GEP-NEN with synchronous liver metastasis and the role of functional status}

Tumor function was available in 1,213 patients (38\%), and these were predominantly of carcinoid type regardless of primary site. Carcinoid GEP-NENs were associated with 
Table 2 Effect of primary tumor site (origin), type and primary treatment modality on survival in GEP-NENs with liver metastasis: unadjusted and adjusted hazard ratios (SEER 2010-2016)

\begin{tabular}{|c|c|c|c|c|c|c|}
\hline \multirow{2}{*}{ Variables } & \multicolumn{6}{|c|}{ Cox regression analysis: GEP-NENs with liver metastasis } \\
\hline & $\mathrm{HR}$ & $P$ value & $\mathrm{HR}$ & $P$ value & $\mathrm{HR}$ & $P$ value \\
\hline \multicolumn{7}{|l|}{ Unadjusted Analysis } \\
\hline \multicolumn{7}{|l|}{ Tumor site (origin) } \\
\hline Pancreas (pNEN) & $1.45(1.3-1.60)$ & $<0.001$ & $0.74(0.55-0.99)$ & 0.041 & $0.91(0.80-1.022)$ & 0.11 \\
\hline Stomach (gNEN) & $2.56(2.12-3.10)$ & $<0.001$ & $1.68(0.92-3.06)$ & 0.092 & $1.63(1.33-2.01)$ & $<0.001$ \\
\hline \multicolumn{7}{|l|}{ Functional type } \\
\hline Carcinoid & 1 (ref) & & 1 (ref) & & 1 (ref) & \\
\hline No & 1 (ref) & & NA & & NA & \\
\hline Yes & $0.29(0.26-0.33)$ & $<0.001$ & NA & & NA & \\
\hline Unknown & $0.71(0.43-1.18)$ & 0.185 & NA & & NA & \\
\hline \multicolumn{7}{|l|}{ Adjusted analysis } \\
\hline \multicolumn{7}{|l|}{ Tumor site (origin) } \\
\hline Intestine (iNEN) & 1 (ref) & & 1 (ref) & & 1 (ref) & \\
\hline Pancreas (pNEN) & $0.92(0.82-1.02)$ & 0.141 & $0.55(0.41-0.75)$ & $<0.001$ & $0.96(0.84-1.09)$ & 0.49 \\
\hline \multicolumn{7}{|l|}{ Surgical resection } \\
\hline No & 1 (ref) & & NA & & NA & \\
\hline Yes & $0.46(0.40-0.53)$ & $<0.001$ & NA & & NA & \\
\hline Unknown & $1.03(0.62-1.71)$ & 0.921 & NA & & NA & \\
\hline
\end{tabular}

GEP-NEN, gastroenteropancreatic neuroendocrine neoplasms; SEER, Surveillance, Epidemiology, and End Results.

better unadjusted survival compared to non-carcinoid tumors. Patients with unspecified tumor types exhibited intermediate survival suggesting that this group includes both carcinoid and non-carcinoid tumors (Figure 5A). The corresponding unadjusted hazard ratios for functional status are summarized in Table 2. Moreover, this pattern of comparatively better carcinoid tumor survival was consistent in case of intestinal, pancreatic and gNENs (Figure 5B,C,D, respectively).

Lastly, carcinoid tumors exhibited different unadjusted survival in case of different primary sites (Figure S2A; $\mathrm{P}<0.001)$ : iNEN (best), pNEN (intermediate) and gNEN (worst). Alternatively, non-carcinoid tumors were associated with similar survival irrespective of tumor origin (Figure S2B; $\mathrm{P}=0.127$ ). The unspecified tumor type (includes 

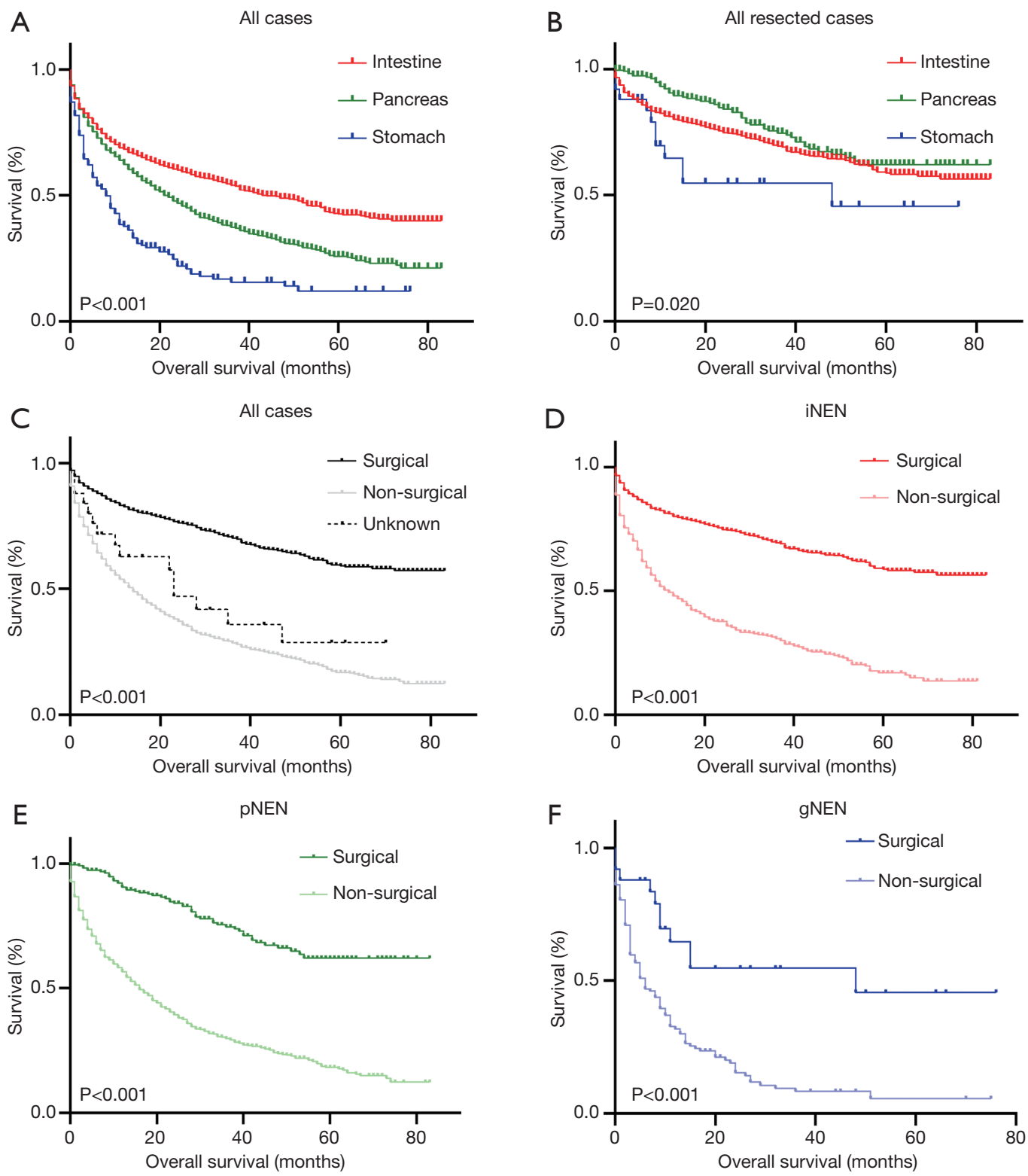

Figure 2 Kaplan-Meier survival curves depicting overall survival in patients with GEP-NENs and synchronous liver metastasis. (A) All patients and (B) all primary resected patients with liver metastatic GEP-NENs based on primary site of origin, (C) all GEP-NENs, (D) iNENs, (E) pNENs and (F) gNENs based on surgical resection. The P values were determined by the log-rank test. GEP-NEN, gastroenteropancreatic neuroendocrine neoplasms.

both types) also showed significance (Figure S2C; $\mathrm{P}<0.001$ ).

\section{Independent effects of primary site, functional status and treatment modality in GEP-NENs with synchronous liver metastasis}

Multivariate risk adjustment was applied to the entire patient cohort (Table 2, Table S1) continued to show gender, age, tumor grade, functional status, primary surgical resection, chemotherapy and synchronous bone or lung metastasis to be independent predictors of survival in addition to primary site [non-carcinoid versus carcinoid: $\operatorname{AHR}(95 \% \mathrm{CI})=3.46(2.18-5.48)]$ and surgical resection $[$ AHR $=0.46(0.40-0.53)]$. Importantly, the GEP-NEN 

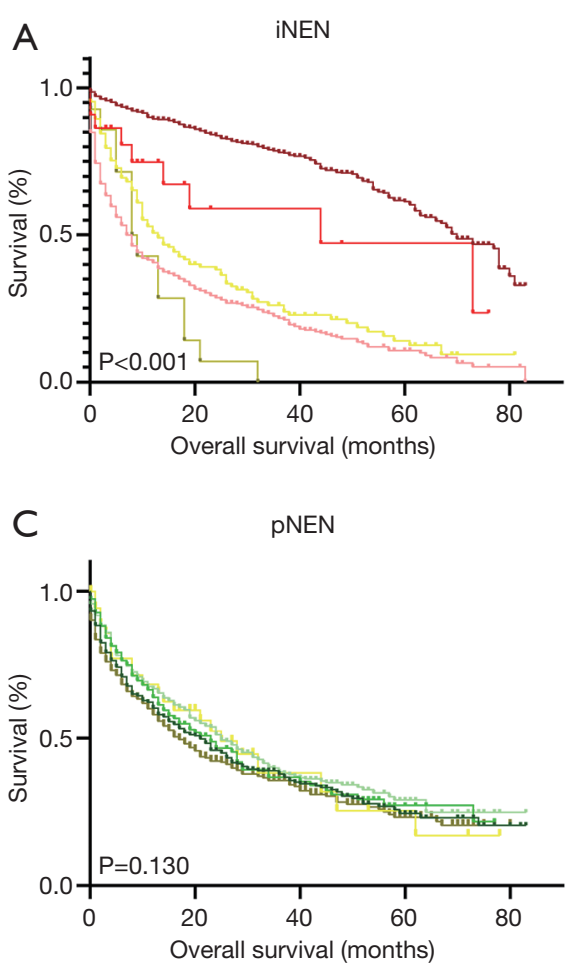

- Small intestine

- Appendix

- Colon

- Rectum

- Anus

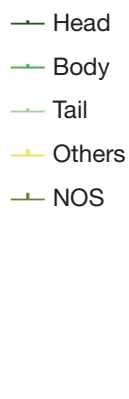

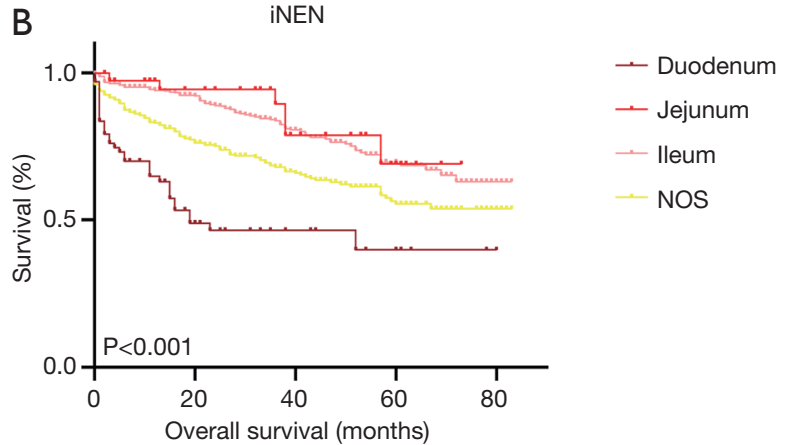

D

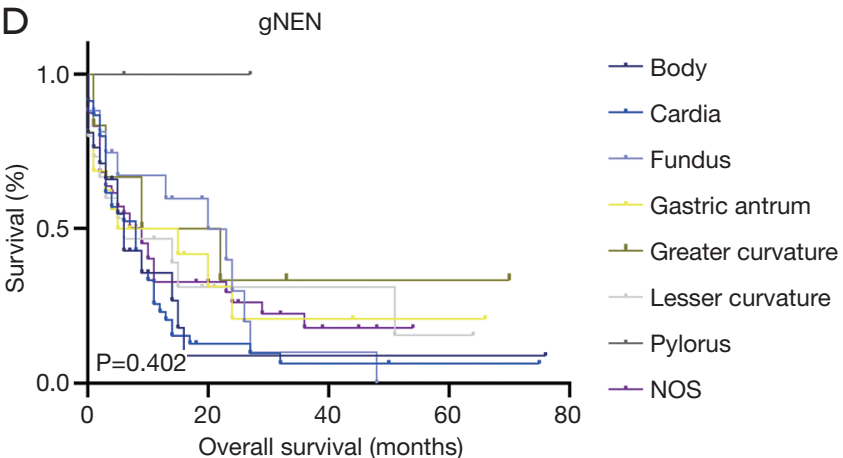

Figure 3 Kaplan-Meier survival curves depicting overall survival in patients with liver metastatic GEP-NENs according to tumor primary sites. $(\mathrm{A}, \mathrm{B})$ iNENs, (C) pNENs and (D) gNENs. The p-values were determined by the log-rank test. GEP-NEN, gastroenteropancreatic neuroendocrine neoplasms.

tumor origin was not independently associated with survival when patients with and without surgical resection were considered collectively.

Separate multivariate analyses of surgical and nonsurgical cases provided further insight on the potential role of primary tumor site. Specifically, in patients treated surgically, comprehensive risk adjustment showed that pNENs $[$ AHR $=0.55(0.41-0.75) ; \mathrm{P}<0.001]$ were associated with better survival than iNENs (reference group). Alternatively, gNENs [AHR =1.75 (0.96-3.21); $\mathrm{P}=0.07$ ] trended towards worse survival than iNENs. Univariable and multivariable analyses were also performed after stratifying by primary tumor site. Briefly, in patients with iNENs, age, tumor grade, functional status and distal tract disease were independent risk factors negatively associated with survival while surgery and chemotherapy were independently associated with improved survival (Table S2). After risk adjustment, patients with pNENs had significantly worse survival if older, with poorer tumor grade, when non-carcinoid, if treated non-surgically or with synchronous bone or lung metastasis (Table S3). In gNENs, only age, functional status and surgery independently predicted survival (Table S4).

\section{Discussion}

GEP-NENs have become more commonly encountered tumors affecting the gastroenteropancreatic system. Yet, their diagnosis remains challenging with patients often presenting late with metastatic disease particularly involving the liver (5). We endeavored to develop accurate models to aid in predicting prognosis in patients of GEP-NENs with metastasis to the liver and, in the process, to elucidate the impact of a variety of patient, clinical and pathologic variables on patient prognosis with particular focus on the roles of tumor origin site, functional status and the primary treatment modality and the interaction between these factors. To do so, we leveraged the national scale US population-based National Cancer Institute SEER program database.

To our knowledge, the analyses on GEP-NENs with synchronous liver metastasis reported in this study are 

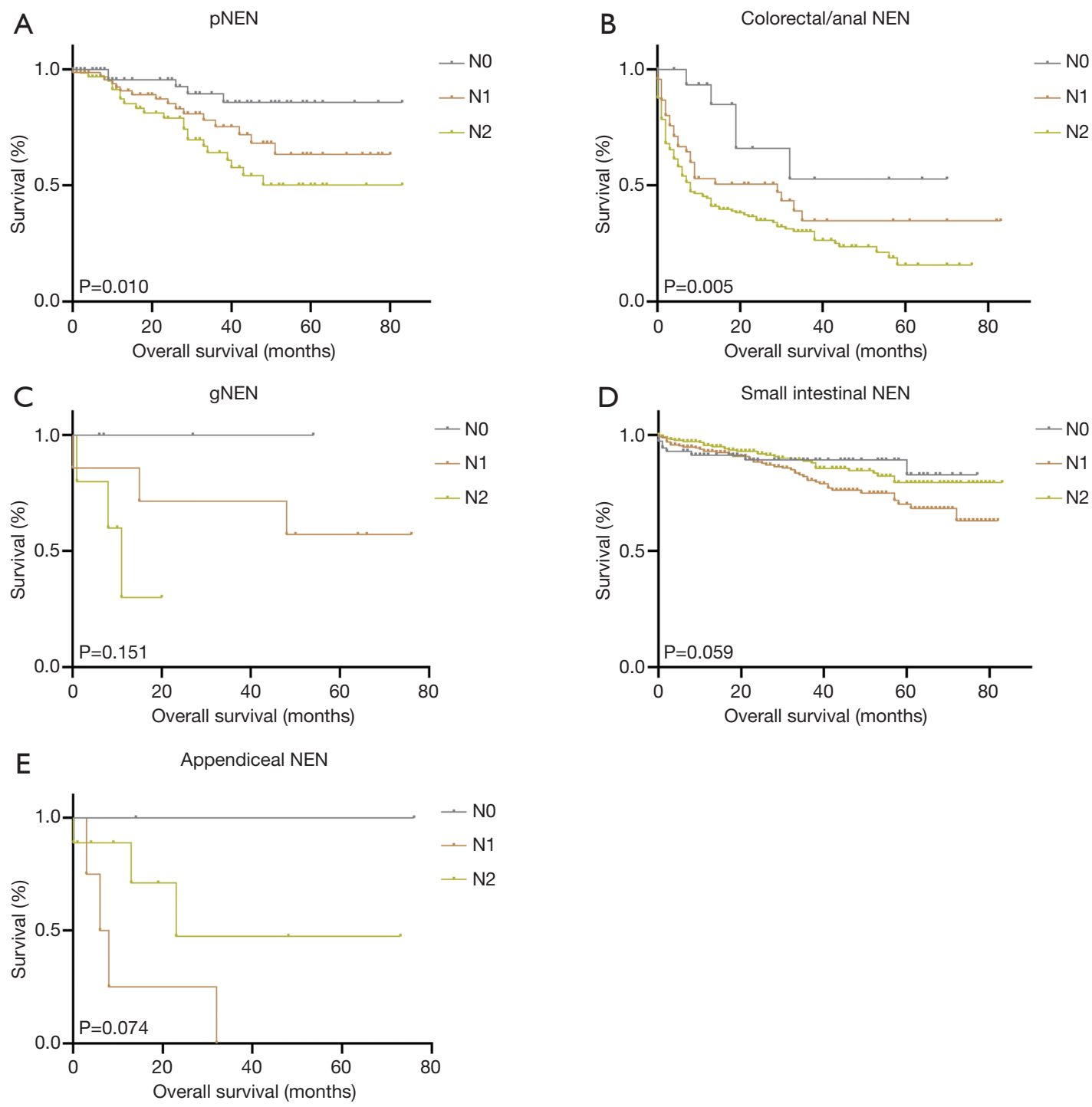

Figure 4 Kaplan-Meier survival curves depicting overall survival in patients with liver metastatic GEP-NENs according to AJCC N staging: (A) pNENs, (B) colorectal/anal NENs, (C) gNENs, (D) small intestinal NENs and (E) appendiceal NENs. The p-values were determined by the log-rank test. GEP-NEN, gastroenteropancreatic neuroendocrine neoplasms.

based on an unprecedented volume of cases that were derived from the national scale SEER database. A number of important findings were gleaned from the current study.

First, GEP-NENs with synchronous liver metastasis have proved to be most commonly of intestinal origin followed by pancreatic and gastric origin. Similar to reports of non-metastatic GEP-NENs, iNENs with liver metastasis were the most common, accounting for 1 in 2 of all cases $(n=1,590)(10,12,13)$. Patients with iNENs and liver metastasis are decidedly more frequently treated with surgical resection compared to both pNENs and
gNENs, and hence the overall survival of iNENs amongst all resected and unresected cases is superior to that in case of pNENs (intermediate) and gNENs (worst). These iNEN patients were shown to have the most favorable outcome with a median of survival of 43 months. However, if primary surgery was performed for GEP-NENs with synchronous liver metastasis, pNEN patients were shown to have the most beneficial survival instead. High grade and non-carcinoid tumors were associated with poorer survival. Additionally, within the intestine, patients with more distal (colorectal) disease and liver metastasis had a significantly 

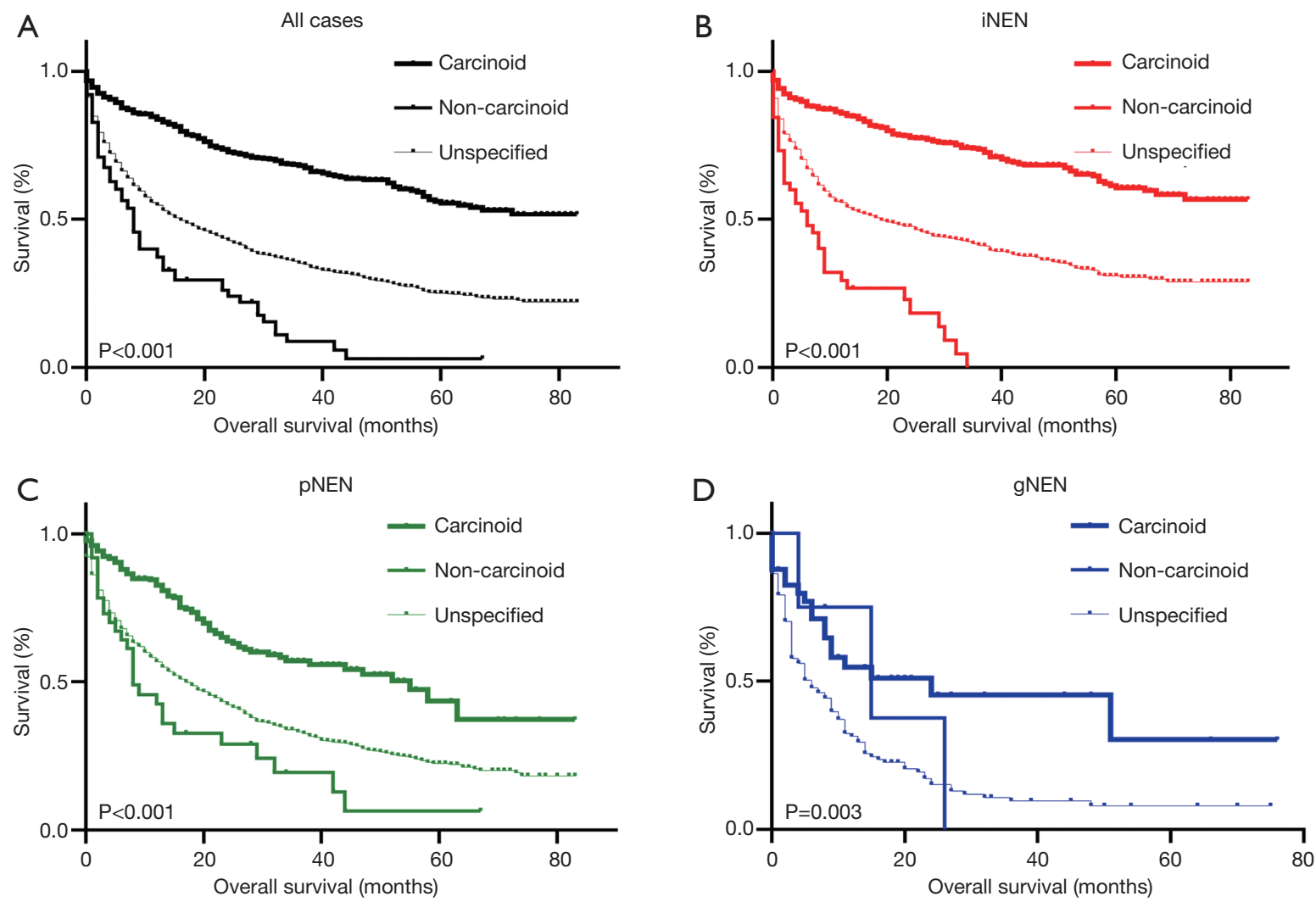

Figure 5 Kaplan-Meier survival curves depicting overall survival in patients with GEP-NENs and synchronous liver metastasis according to functional status. (A) All GEP-NENs, (B) iNENs, (C) pNENs and (D) gNENs. The p-values were determined by the log-rank test.

worse prognosis than those with more proximal (small intestine) originating iNENs with synchronous liver metastasis. This may be partially attributed to a significantly increased lymph node involvement in colorectal and anal NENs (LNR 0.5) as compared to that of the small intestine (LNR 0.3). These data may indicate more aggressive tumor biology and delayed diagnosis in colorectal and anal NENs.

In case of localized, non-metastatic GEP-NENs, pancreatic originating NENs are associated with the poorest survival followed by gastric and then intestinal (14). Pancreatic-originating NENs were the second most common source of liver metastatic GEP-NEN ( $n=1,427)$ accounting for almost $45 \%$ of cases. This is consistent with findings from another population-based study by Gudmundsdottir and colleagues (13). These exhibited a worse unadjusted overall survival than iNENs with an almost halved median survival of 22 (CI, 19.4-24.6) months. The majority of these presented in the tail (30.69\%) followed by the head (28.66\%) and body (10.86\%). However, location within the pancreas showed no significant association with survival. This is contrary to findings in pancreatic ductal adenocarcinoma (PDAC), the most common type of pancreatic cancer, which has a more favorable prognosis when located in the head of the pancreas (15). Similar to colorectal cancer, there has also been consideration for surgical treatment in oligometastatic pancreatic cancer when confined to the liver for highly selected patients (16). Here, for pNENs, both radiotherapy and chemotherapy showed no improvement in survival while surgery provided a pronounced enhancement in survival. Although treating metastatic disease with surgical intervention is seldom a standard, this finding draws comparisons to results that have led to the NCCN guidelines regarding surgical intervention for colorectal cancer with oligometastatic liver disease (17). Our current analyses support this therapeutic option in NEN with synchronous liver metastasis as well.

Of all GEP-NENs, primary gastric and rectal NENs have been shown to have the least metastatic potential $(13,18)$. This may partly explain the low number of gNENs 
with liver metastasis available for the current study $(\mathrm{n}=171)$. These were associated with the poorest outcomes displaying a dismal 8-month overall median survival underscoring the importance of diagnosing gNENs in a pre-metastatic stage to avoid extremely poor outcomes. While less frequent, gNENs with liver metastasis appear to be associated with a more aggressive tumor biology and comparatively greater LNR compared to pNENs and hence leading to poorer survival.

Second, surgical treatment of GEP-NENs was associated with substantially better survival compared to non-surgically treated GEP-NENs irrespective of the primary tumor origin. Alternatively, primary tumor site was found to be an independent prognostic factor in case of surgical resection [pNENs (best) and gNENs (worst)] but not for nonsurgical patients. Interestingly, after risk adjustment, GEPNENs with synchronous liver metastasis treated by surgery showed more favorable survival across all tumor origins. This finding is consistent with other reports in the literature (19-21). Amongst the resected group, pNENs displayed a better response to surgical intervention than iNENs which too tended to respond more favorably than gNENs although statistical significance was not reached. This could be partially explained by lack of power since only 25 patients with gNENs and liver metastasis were treated by surgery. It is noteworthy that both surgery and adjunct chemotherapy were found to be independently associated with improved survival. Radiotherapy showed no significant improvement.

Third, GEP-NENs of carcinoid tumor type were more frequently treated with surgical resection and were consequently associated with better survival regardless of primary tumor site. Across all GEP-NENs with synchronous liver metastasis, carcinoid NENs were associated with better survival compared to non-carcinoid NENs. This finding is consistent with the literature on non-metastatic GEP-NENs as carcinoid tumors are often diagnosed earlier due to their unique presenting symptoms and are associated with an 83\% 5-year overall survival when surgically treated (22).

The three major GEP-NEN classification systems that are widely used include (I) the World Health Organization (WHO) classification (23); (II) the Union for International Cancer Control TNM system (24); (III) the European NEN Society TNM grading system (25). Each staging system presents advantages and disadvantages and perhaps a standardized tool that integrates the best aspects of each would provide the most accurate staging and prognostic information $(26,27)$. In light of our and others' findings that support more aggressive surgical intervention, we sought out to investigate any additional prognostic value by applying the post-surgical $\mathrm{N}$ AJCC $8^{\text {th }}$ edition staging system (28-30). In fact the new $8^{\text {th }}$ edition amendment to the $\mathrm{N}$ classification of the AJCC TNM staging system showed a significant discrimination in pancreatic and colorectal/anal NEN with synchronous liver metastasis. Perhaps a dynamic risk stratification unifying the above pre- and post-surgical staging systems would provide the optimal risk stratification. This may be an especially timely consideration given that patients are increasingly receiving surgical treatment of GEP-NENs with synchronous liver metastasis.

\section{Limitations}

This population-based study was performed using the nationally representative SEER database. However, limitations persist. First, the retrospective and national scale nature of the study led to the inherent limitation of possible selection bias due to excluded patients with incomplete and unrecoverable data. Additionally, the SEER program does not report Ki-67 index which is an important measure for NEN aggressiveness in the WHO classification system. Furthermore, detailed data are not reported regarding certain treatments that may impact survival. For example, these may include resection margin status or type, time and duration of chemotherapy. Moreover, it is important to consider that the performance status of patients was not known, and it is possible that some patients were too sick to tolerate surgery. Nevertheless, the large sample database mitigates concerns about generalizability, the study power needed to explore rarer diseases and allows investigators to probe hypothesis driven queries that are otherwise difficult. Based on our results, future randomized prospective studies should investigate and validate our findings.

\section{Conclusions}

Our study confirms the superiority of surgical resection for the treatment of GEP-NENs with synchronous liver metastasis irrespective of primary tumor site. We also uncovered a notable variance of outcomes of this specific sub-cohort of GEP-NEN patients specifically with functional status as well as the intra organ primary tumor location in case of intestinal NENs. 


\section{Acknowledgments}

Funding: This work was funded by the National Natural Science Foundation of China (81773068).

\section{Footnote}

Reporting Checklist: The authors have completed the STROBE reporting checklist. Available at http://dx.doi. org/10.21037/atm-20-5348

Conflicts of Interest: All authors have completed the ICMJE uniform disclosure form (available at http://dx.doi. org/10.21037/atm-20-5348). The authors have no conflicts of interest to declare.

Ethical Statement: The authors are accountable for all aspects of the work in ensuring that questions related to the accuracy or integrity of any part of the work are appropriately investigated and resolved. The study was conducted in accordance with the Declaration of Helsinki (as revised in 2013). The study was approved by institutional ethics committee of Zhongshan Hospital, Fudan University (No. Y2020.094) and individual consent was waived due to the retrospective nature of the analysis.

Open Access Statement: This is an Open Access article distributed in accordance with the Creative Commons Attribution-NonCommercial-NoDerivs 4.0 International License (CC BY-NC-ND 4.0), which permits the noncommercial replication and distribution of the article with the strict proviso that no changes or edits are made and the original work is properly cited (including links to both the formal publication through the relevant DOI and the license). See: https://creativecommons.org/licenses/by-nc-nd/4.0/.

\section{References}

1. Barakat MT, Meeran K, Bloom SR. Neuroendocrine tumours. Endocr Relat Cancer 2004;11:1-18.

2. Dasari A, Shen C, Halperin D, et al. Trends in the Incidence, Prevalence, and Survival Outcomes in Patients With Neuroendocrine Tumors in the United States. JAMA Oncol 2017;3:1335-42.

3. Yao JC, Hassan M, Phan A, et al. One hundred years after "carcinoid": epidemiology of and prognostic factors for neuroendocrine tumors in 35,825 cases in the United States. J Clin Oncol 2008;26:3063-72.
4. Niederle MB, Hackl M, Kaserer K, et al. Gastroenteropancreatic neuroendocrine tumours: the current incidence and staging based on the WHO and European Neuroendocrine Tumour Society classification: an analysis based on prospectively collected parameters. Endocr Relat Cancer 2010;17:909-18.

5. Modlin IM, Oberg K, Chung DC, et al. Gastroenteropancreatic neuroendocrine tumours. Lancet Oncol 2008;9:61-72.

6. Bhosale P, Shah A, Wei W, et al. Carcinoid tumours: predicting the location of the primary neoplasm based on the sites of metastases. Eur Radiol 2013;23:400-7.

7. Riihimäki M, Hemminki A, Sundquist K, et al. The epidemiology of metastases in neuroendocrine tumors. Int J Cancer 2016;139:2679-86.

8. Modlin IM, Lye KD, Kidd M. A 5-decade analysis of 13,715 carcinoid tumors. Cancer 2003;97:934-59.

9. Pape UF, Berndt U, Muller-Nordhorn J, et al. Prognostic factors of long-term outcome in gastroenteropancreatic neuroendocrine tumours. Endocr Relat Cancer 2008;15:1083-97.

10. Garcia-Carbonero R, Capdevila J, Crespo-Herrero G, et al. Incidence, patterns of care and prognostic factors for outcome of gastroenteropancreatic neuroendocrine tumors (GEP-NETs): results from the National Cancer Registry of Spain (RGETNE). Ann Oncol 2010;21:1794-803.

11. Pu N, Yin L, Habib JR, et al. Optimized modification of the eighth edition of AJCC TNM staging system for resected pancreatic ductal adenocarcinoma. Future Oncol 2019;15:3457-65.

12. Frilling A, Akerstrom G, Falconi M, et al. Neuroendocrine tumor disease: an evolving landscape. Endocr Relat Cancer 2012;19:R163-85.

13. Gudmundsdottir H, Moller PH, Jonasson JG, et al. Gastroenteropancreatic neuroendocrine tumors in Iceland: a population-based study. Scand J Gastroenterol 2019;54:69-75.

14. Man D, Wu J, Shen Z, et al. Prognosis of patients with neuroendocrine tumor: a SEER database analysis. Cancer Manag Res 2018;10:5629-38.

15. Artinyan A, Soriano PA, Prendergast C, et al. The anatomic location of pancreatic cancer is a prognostic factor for survival. HPB (Oxford) 2008;10:371-6.

16. Hackert T, Niesen W, Hinz U, et al. Radical surgery of oligometastatic pancreatic cancer. Eur J Surg Oncol 2017;43:358-63.

17. Benson AB 3rd, Choti MA, Cohen AM, et al. NCCN Practice Guidelines for Colorectal Cancer. Oncology 
(Williston Park) 2000;14:203-12.

18. Hallet J, Law CH, Cukier M, et al. Exploring the rising incidence of neuroendocrine tumors: a population-based analysis of epidemiology, metastatic presentation, and outcomes. Cancer 2015;121:589-97.

19. Norton JA, Warren RS, Kelly MG, et al. Aggressive surgery for metastatic liver neuroendocrine tumors. Surgery 2003;134:1057-63; discussion 1063-5.

20. Sarmiento JM, Heywood G, Rubin J, et al. Surgical treatment of neuroendocrine metastases to the liver: a plea for resection to increase survival. J Am Coll Surg 2003;197:29-37.

21. Elias D, Lasser P, Ducreux M, et al. Liver resection (and associated extrahepatic resections) for metastatic welldifferentiated endocrine tumors: a 15-year single center prospective study. Surgery 2003;133:375-82.

22. Shebani KO, Souba WW, Finkelstein DM, et al. Prognosis and survival in patients with gastrointestinal tract carcinoid tumors. Ann Surg 1999;229:815-21; discussion 822-3.

23. Capelli P, Fassan M, Scarpa A. Pathology - grading and staging of GEP-NETs. Best Pract Res Clin Gastroenterol 2012;26:705-17.

24. Sobin L, Gospodarowicz M, Wittekind C. TNM classification of malignant tumours.7th ed. Oxford: Wiley-

Cite this article as: $\mathrm{Pu} \mathrm{N}$, Habib JR, Bejjani M, Yin H, Nagai M, Chen J, Kinny-Köster B, Chen Q, Zhang J, Yu J, $\mathrm{Wu} \mathrm{W}$, Lou $\mathrm{W}$. The effect of primary site, functional status and treatment modality on survival in gastroenteropancreatic neuroendocrine neoplasms with synchronous liver metastasis: a US population-based study. Ann Transl Med 2021;9(4):329. doi: 10.21037/atm-20-5348
Blackwell, 2010.

25. Rindi G, Kloppel G, Alhman H, et al. TNM staging of foregut (neuro)endocrine tumors: a consensus proposal including a grading system. Virchows Arch 2006;449:395-401.

26. Díez M, Teulé A, Salazar R. Gastroenteropancreatic neuroendocrine tumors: diagnosis and treatment. Ann Gastroenterol 2013;26:29-36.

27. Gao S, Pu N, Liu L, et al. The latest exploration of staging and prognostic classification for pancreatic neuroendocrine tumors: a large population-based study. Journal of Cancer 2018;9:1698-706.

28. Kakar S, Pawlik TM, Allen PJ, et al. Exocrine pancreas. Pancreatic adenocarcinoma. In: AJCC Cancer Staging Manual (8th Edition). New York: Springer-Verlag, 2016.

29. Gurusamy KS, Ramamoorthy R, Sharma D, et al. Liver resection versus other treatments for neuroendocrine tumours in patients with resectable liver metastases. Cochrane Database Syst Rev 2009;2009:CD007060.

30. Pavel M, Baudin E, Couvelard A, et al. ENETS Consensus Guidelines for the management of patients with liver and other distant metastases from neuroendocrine neoplasms of foregut, midgut, hindgut, and unknown primary. Neuroendocrinology 2012;95:157-76. 


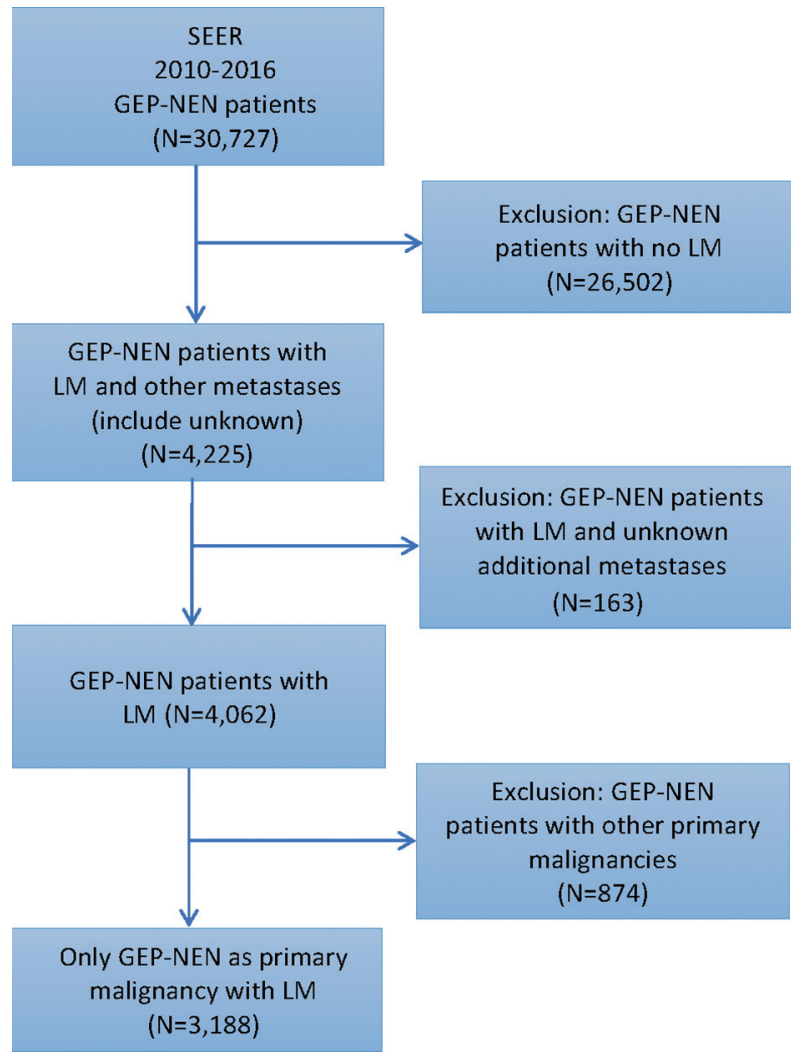

Figure S1 Flowchart of the inclusion and exclusion criteria.
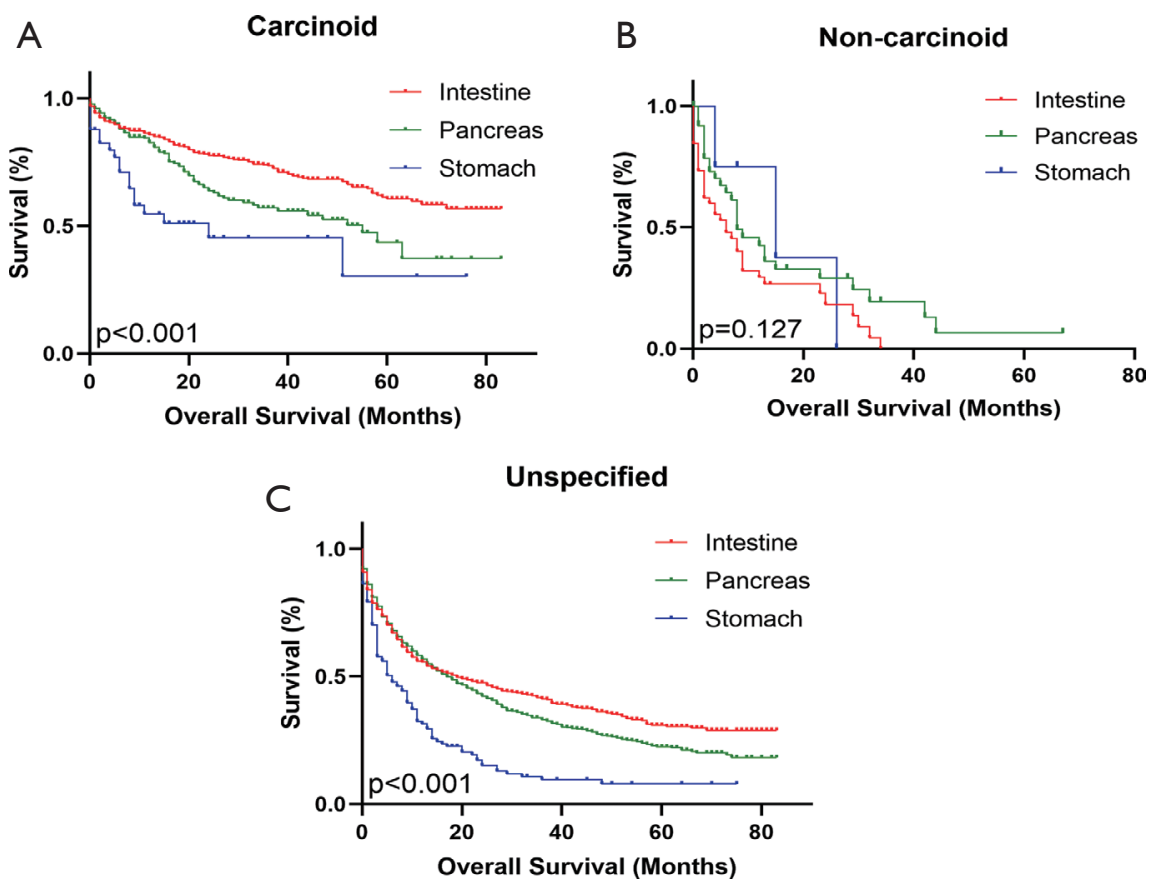

Figure S2 Kaplan-Meier survival curves depicting overall survival in patients with liver metastatic GEP-NENs according to tumor primary sites among different functional status: (A) carcinoid, (B) non-carcinoid, and (C) unspecified tumor type (includes both types). The p-values were determined by the log-rank test. 
Table S1 Demographic and disease factors in all primary GEP-NEN patients with liver metastasis (all origin locations) - univariate and multivariate Cox regression results

\begin{tabular}{|c|c|c|c|c|c|}
\hline Variables & $\begin{array}{l}\text { Patients } \\
(n=3188)\end{array}$ & \multicolumn{4}{|c|}{ Cox regression analysis } \\
\hline Female & 1444 & Reference & $1.151(1.043-1.269)$ & Reference & 1 \\
\hline \multicolumn{6}{|l|}{ Age, years } \\
\hline Average, range & $60.9(4-97)$ & $<0.001$ & $1.031(1.027-1.035)$ & $<0.001$ & $1.024(1.020-1.028)$ \\
\hline \multicolumn{6}{|l|}{ Tumor site } \\
\hline Intestine & 1590 & Reference & 1 & Reference & 1 \\
\hline well & 906 & Reference & 1 & Reference & 1 \\
\hline Moderate & 394 & 0.011 & 1.337 (1.069-1.673) & 0.041 & $1.264(1.010-1.583)$ \\
\hline Poor or undifferentiated & 621 & $<0.001$ & $7.625(6.494-8.952)$ & $<0.001$ & $4.755(3.977-5.686)$ \\
\hline Unknown & 1267 & $<0.001$ & $3.544(3.052-4.115)$ & $<0.001$ & $2.163(1.837-2.547)$ \\
\hline \multicolumn{6}{|l|}{ Functional type } \\
\hline Carcinoid tumor & 1125 & Reference & 1 & Reference & 1 \\
\hline Non-carcinoid tumor & 88 & $<0.001$ & $4.611(3.541-6.003)$ & $<0.001$ & $2.633(2.006-3.456)$ \\
\hline \multicolumn{6}{|l|}{ Surgery } \\
\hline \multicolumn{6}{|l|}{ Radiotherapy } \\
\hline No & 2975 & Reference & $1.357(1.135-1.622)$ & Reference & 1 \\
\hline Yes & 213 & 0.001 & & 0.428 & $1.078(0.895-1.298)$ \\
\hline \multicolumn{6}{|l|}{ Chemotherapy } \\
\hline No & 1888 & Reference & $1.472(1.335-1.623)$ & Reference & 1 \\
\hline Yes & 1300 & $<0.001$ & & $<0.001$ & $0.820(0.734-0.916)$ \\
\hline \multicolumn{6}{|l|}{ Bone metastasis } \\
\hline No & 2953 & Reference & 2.064 (1.758-2.422) & Reference & 1 \\
\hline Yes & 235 & $<0.001$ & & 0.007 & $1.261(1.067-1.491)$ \\
\hline \multicolumn{6}{|l|}{ Brain metastasis } \\
\hline No & 3163 & Reference & $3.183(2.046-4.954)$ & Reference & 1 \\
\hline Yes & 25 & $<0.001$ & & 0.647 & $0.897(0.564-1.428)$ \\
\hline \multicolumn{6}{|l|}{ Lung metastasis } \\
\hline No & 2944 & Reference & $2.506(2.147-2.926)$ & Reference & 1 \\
\hline Yes & 244 & $<0.001$ & & $<0.001$ & $1.483(1.260-1.745)$ \\
\hline
\end{tabular}

(c) Annals of Translational Medicine. All rights reserved. 
Table S2 Demographic and disease factors in all primary iNEN patients with liver metastasis - univariate and multivariate Cox regression results

\begin{tabular}{|c|c|c|c|c|c|}
\hline Variables & $\begin{array}{l}\text { Patients } \\
(n=1590)\end{array}$ & \multicolumn{4}{|c|}{ Overall survival } \\
\hline Female & 773 & Reference & 1 & & \\
\hline Male & 817 & 0.086 & $1.139(0.982-1.321)$ & & \\
\hline Average, range & $61.2,14-95$ & $<0.001$ & $1.035(1.028-1.041)$ & $<0.001$ & $1.033(1.027-1.040)$ \\
\hline \multicolumn{6}{|l|}{ Grade } \\
\hline Well & 597 & Reference & & Reference & 1 \\
\hline \multicolumn{6}{|l|}{ Functional type } \\
\hline Carcinoid tumor & 726 & Reference & 1 & Reference & 1 \\
\hline Non- carcinoid tumor & 45 & $<0.001$ & $6.246(4.372-8.922)$ & $<0.001$ & $2.138(1.461-3.128)$ \\
\hline Unspecified neuroendocrine tumor & 819 & $<0.001$ & $2.789(2.350-3.309)$ & $<0.001$ & $1.722(1.427-2.078)$ \\
\hline \multicolumn{6}{|l|}{ Site } \\
\hline Colorectum and anus & 625 & Reference & 1 & Reference & 1 \\
\hline Appendix & 22 & 0.008 & $0.409(0.211-0.791)$ & 0.120 & $0.586(0.299-1.150)$ \\
\hline \multicolumn{6}{|l|}{ Surgery } \\
\hline \multicolumn{6}{|l|}{ Radiotherapy } \\
\hline No & 1492 & Reference & 1 & Reference & 1 \\
\hline Yes & 98 & 0.002 & $1.531(1.170-2.003)$ & 0.867 & $1.024(0.774-1.355)$ \\
\hline \multicolumn{6}{|l|}{ Chemotherapy } \\
\hline No & 1117 & Reference & 1 & Reference & 1 \\
\hline Yes & 473 & $<0.001$ & $1.908(1.641-2.219)$ & 0.004 & $0.759(0.630-0.915)$ \\
\hline \multicolumn{6}{|l|}{ Bone metastasis } \\
\hline No & 1490 & Reference & 1 & Reference & 1 \\
\hline Yes & 100 & $<0.001$ & 2.587 (2.023-3.308) & 0.717 & $0.953(0.733-1.238)$ \\
\hline \multicolumn{6}{|l|}{ Brain metastasis } \\
\hline No & 1579 & Reference & 1 & Reference & 1 \\
\hline Yes & 11 & 0.001 & $3.304(1.643-6.644)$ & 0.691 & $1.160(0.558-2.408)$ \\
\hline \multicolumn{6}{|l|}{ Lung metastasis } \\
\hline No & 1477 & Reference & 1 & Reference & 1 \\
\hline Yes & 113 & $<0.001$ & $2.604(2.065-3.285)$ & 0.052 & $1.273(0.998-1.624)$ \\
\hline
\end{tabular}

(C) Annals of Translational Medicine. All rights reserved. 
Table S3 Demographic and disease factors in all primary pNEN patients with liver metastasis - univariate and multivariate Cox regression results

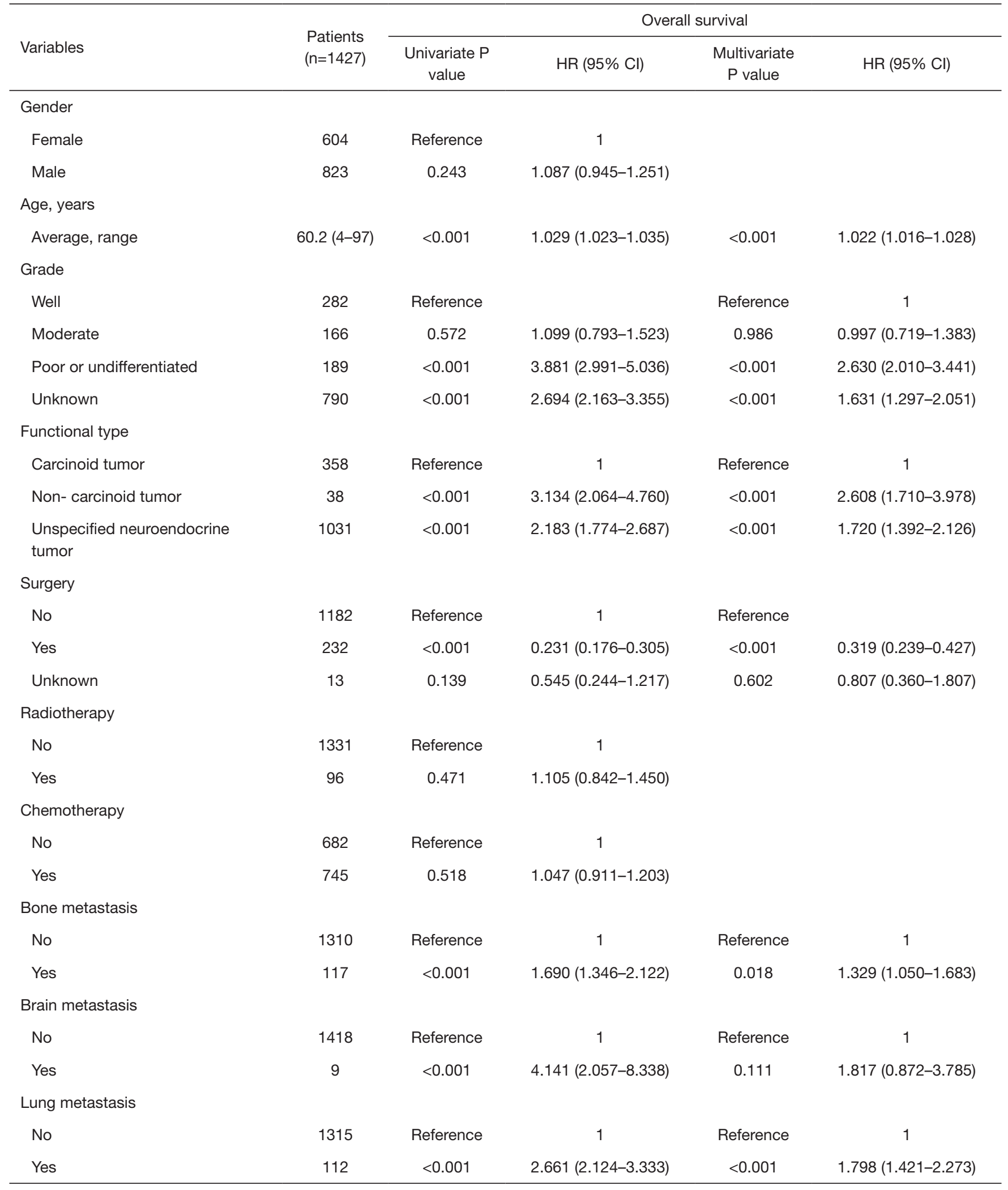


Table S4 Demographic and disease factors in all primary gNEN patients with liver metastasis - univariate and multivariate Cox regression results

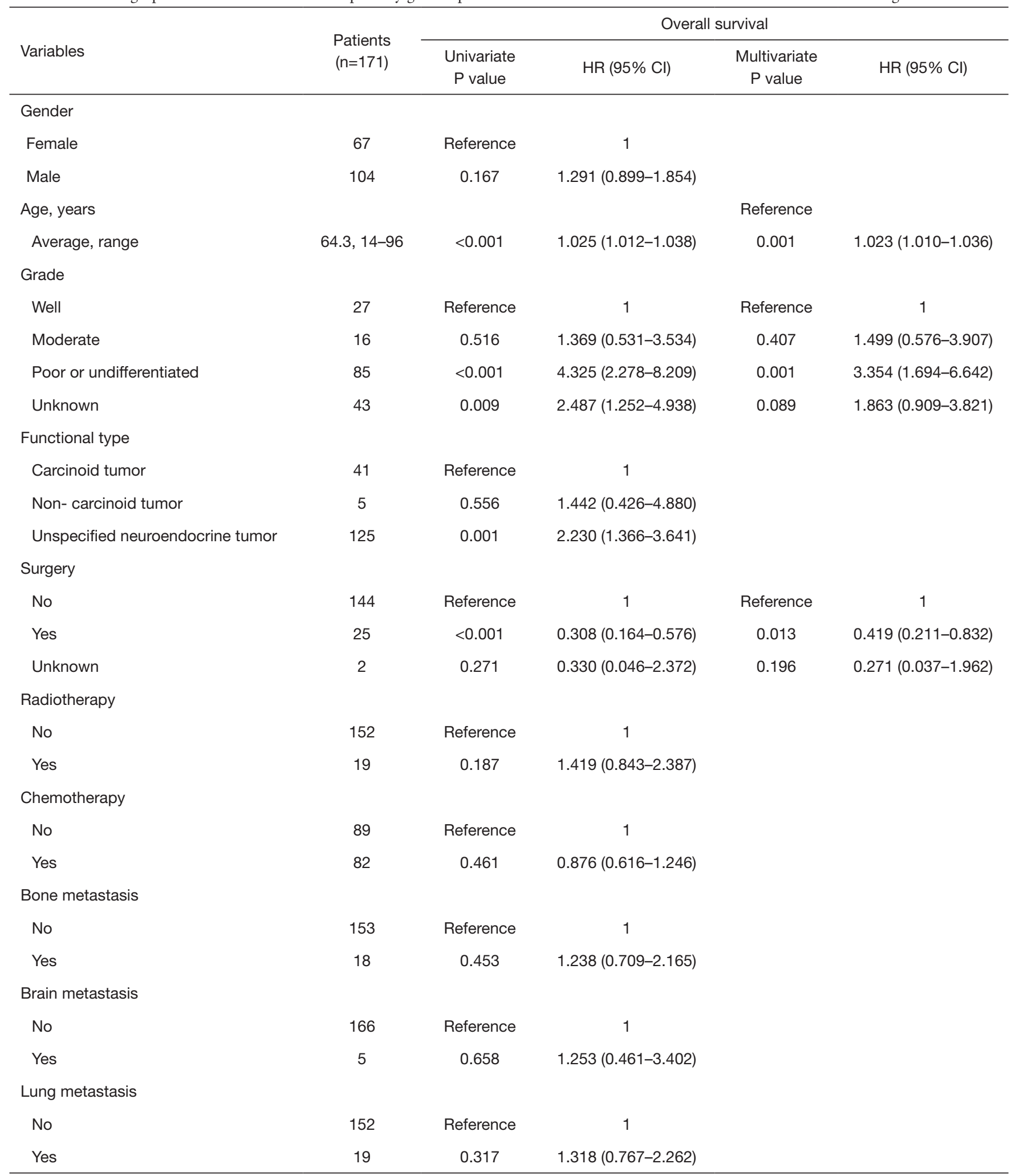

